\title{
BMJ Open Interrupting transmission of soil- transmitted helminths: a study protocol for cluster randomised trials evaluating alternative treatment strategies and delivery systems in Kenya
}

Simon J Brooker, ${ }^{1}$ Charles S Mwandawiro, ${ }^{2}$ Katherine E Halliday, ${ }^{1}$ Sammy M Njenga, ${ }^{2}$ Carlos Mcharo, ${ }^{3}$ Paul M Gichuki, ${ }^{2}$ Beatrice Wasunna, ${ }^{2}$ Jimmy H Kihara, ${ }^{2}$ Doris Njomo, ${ }^{2}$ Dorcas Alusala, ${ }^{4}$ Athuman Chiguzo, ${ }^{5}$ Hugo C Turner, ${ }^{6}$ Caroline Teti, ${ }^{3}$ Claire Gwayi-Chore, ${ }^{3}$ Birgit Nikolay, ${ }^{1}$ James E Truscott, ${ }^{6}$ T Déirdre Hollingsworth, ${ }^{7,8}$ Dina Balabanova, ${ }^{9}$ Ulla K Griffiths, ${ }^{9}$ Matthew C Freeman, ${ }^{10}$ Elizabeth Allen, ${ }^{11}$ Rachel L Pullan, ${ }^{1}$ Roy M Anderson ${ }^{6}$

To cite: Brooker SJ, Mwandawiro CS, Halliday KE, et al. Interrupting transmission of soil-transmitted helminths: a study protocol for cluster randomised trials evaluating alternative treatment strategies and delivery systems in Kenya. BMJ Open 2015;5:e008950. doi:10.1136/bmjopen-2015008950

- Prepublication history for this paper is available online. To view these files please visit the journal online (http://dx.doi.org/10.1136/ bmjopen-2015-008950).

Received 1 June 2015 Accepted 30 July 2015

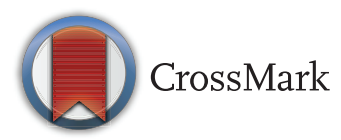

For numbered affiliations see end of article.

Correspondence to Dr Rachel Pullan; rachel.pullan@Ishtm.ac.uk

\section{ABSTRACT}

Introduction: In recent years, an unprecedented emphasis has been given to the control of neglected tropical diseases, including soil-transmitted helminths (STHs). The mainstay of STH control is school-based deworming (SBD), but mathematical modelling has shown that in all but very low transmission settings, SBD is unlikely to interrupt transmission, and that new treatment strategies are required. This study seeks to answer the question: is it possible to interrupt the transmission of STH, and, if so, what is the most costeffective treatment strategy and delivery system to achieve this goal?

Methods and analysis: Two cluster randomised trials are being implemented in contrasting settings in Kenya. The interventions are annual mass anthelmintic treatment delivered to preschool- and school-aged children, as part of a national SBD programme, or to entire communities, delivered by community health workers. Allocation to study group is by cluster, using predefined units used in public health provisiontermed community units (CUs). CUs are randomised to one of three groups: receiving either (1) annual SBD;

(2) annual community-based deworming (CBD); or (3) biannual CBD. The primary outcome measure is the prevalence of hookworm infection, assessed by four cross-sectional surveys. Secondary outcomes are prevalence of Ascaris lumbricoides and Trichuris trichiura, intensity of species infections and treatment coverage. Costs and cost-effectiveness will be evaluated. Among a random subsample of participants, worm burden and proportion of unfertilised eggs will be assessed longitudinally. A nested process evaluation, using semistructured interviews, focus group discussions and a stakeholder analysis, will investigate the community acceptability, feasibility and scale-up of each delivery system.

\section{Strengths and limitations of this study}

- The study has a strong design incorporating random allocation, blinding of assessors to the primary outcome, and builds on and will, subsequently, refine mathematical modelling.

- The interventions include alternative treatment strategies using two different delivery systems, and are well-established, of long duration (24 months) and nested within an ongoing national control programme.

- The study includes cost-effectiveness analysis and analysis of community acceptability, feasibility and scale-up of each delivery system.

- A limitation of the study is its reliance on existing health structures to implement the intervention.

- The study will contribute to evidence regarding the cost-effectiveness of soil-transmitted helminth control and thereby inform national and global policy.

Ethics and dissemination: Study protocols have been reviewed and approved by the ethics committees of the Kenya Medical Research Institute and National Ethics Review Committee, and London School of Hygiene and Tropical Medicine. The study has a dedicated web site.

Trial registration number: NCT02397772.

\section{INTRODUCTION}

Neglected tropical diseases (NTDs) are a cluster of tropical diseases that affect more than one billion people worldwide, mainly 
Figure 1 Summary of study design.

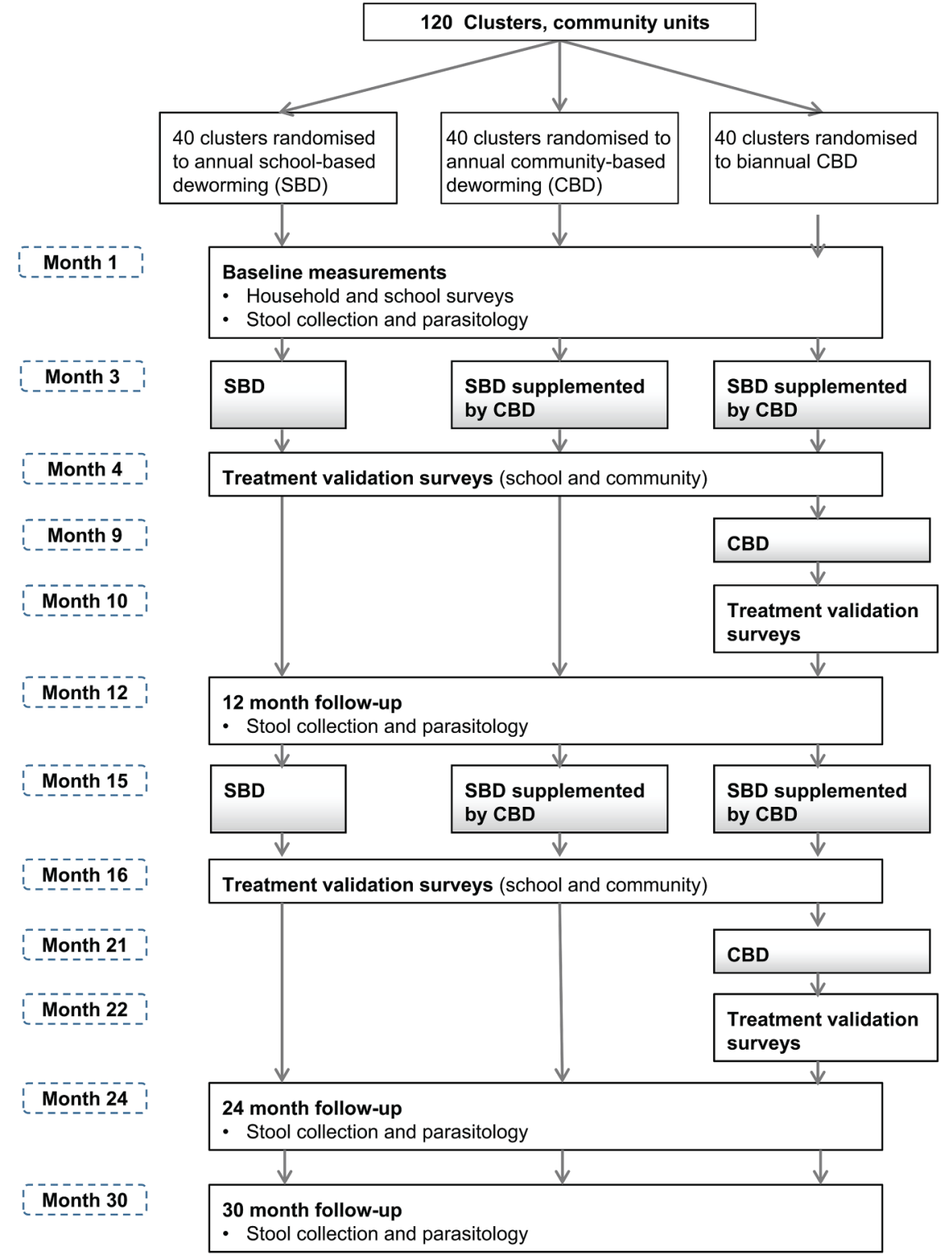

among poor populations living at the periphery of health systems. ${ }^{1}$ NTDs can cause disability, disfigurement, undernutrition and cognitive impairment, yet many NTDs can be easily controlled by periodic mass treatment using safe and broad spectrum drugs. Global efforts to control NTDs reached a turning point in 2012, when WHO launched its NTD Roadmap, ${ }^{1}$ and partners met in London, and pledged to work together to control and eliminate 10 NTDs by $2020 .^{2}$ As part of this commitment, pharmaceutical companies pledged to donate the drugs required for mass treatment programmes and the challenge now is to support countries in developing sustainable systems to distribute donated medicines.

According to the 2010 Global Burden of Disease study, ${ }^{2}$ the soil-transmitted helminths (STHs) spp Ascaris lumbricoides, Trichuris trichiura and hookworm, contribute the greatest disease burden among the NTDs, causing an estimated 4.98 million years lived with disability each year. ${ }^{3}$ Fortunately, much of this burden can be readily averted by periodic, population-based chemotherapy (also known as deworming). The WHO identifies three priority groups for deworming: school-age children, preschool-age children and women of childbearing age, ${ }^{4}$ as they typically harbour chronic and intense infections at a time when they are undergoing physical and/or cognitive development. An effective mechanism to reach school-age children is provided by school-based deworming programmes, which have been shown to costeffectively reduce their STH-related morbidity. ${ }^{5} 6$ In 2013, some 237 million school-age children-equivalent to $39 \%$ of the global at-risk school-aged populationbenefitted from STH treatment. ${ }^{7}$ However, if the 2020 target of treating $75 \%$ of school-age children is to be reached, there needs to be a concerted effort to scale-up deworming. Responding to this need, a new consortium was established in Paris in 2014, to assist countries to develop mechanisms for addressing STH among 
Figure 2 Map showing location of study sites and community units.
Bungoma County

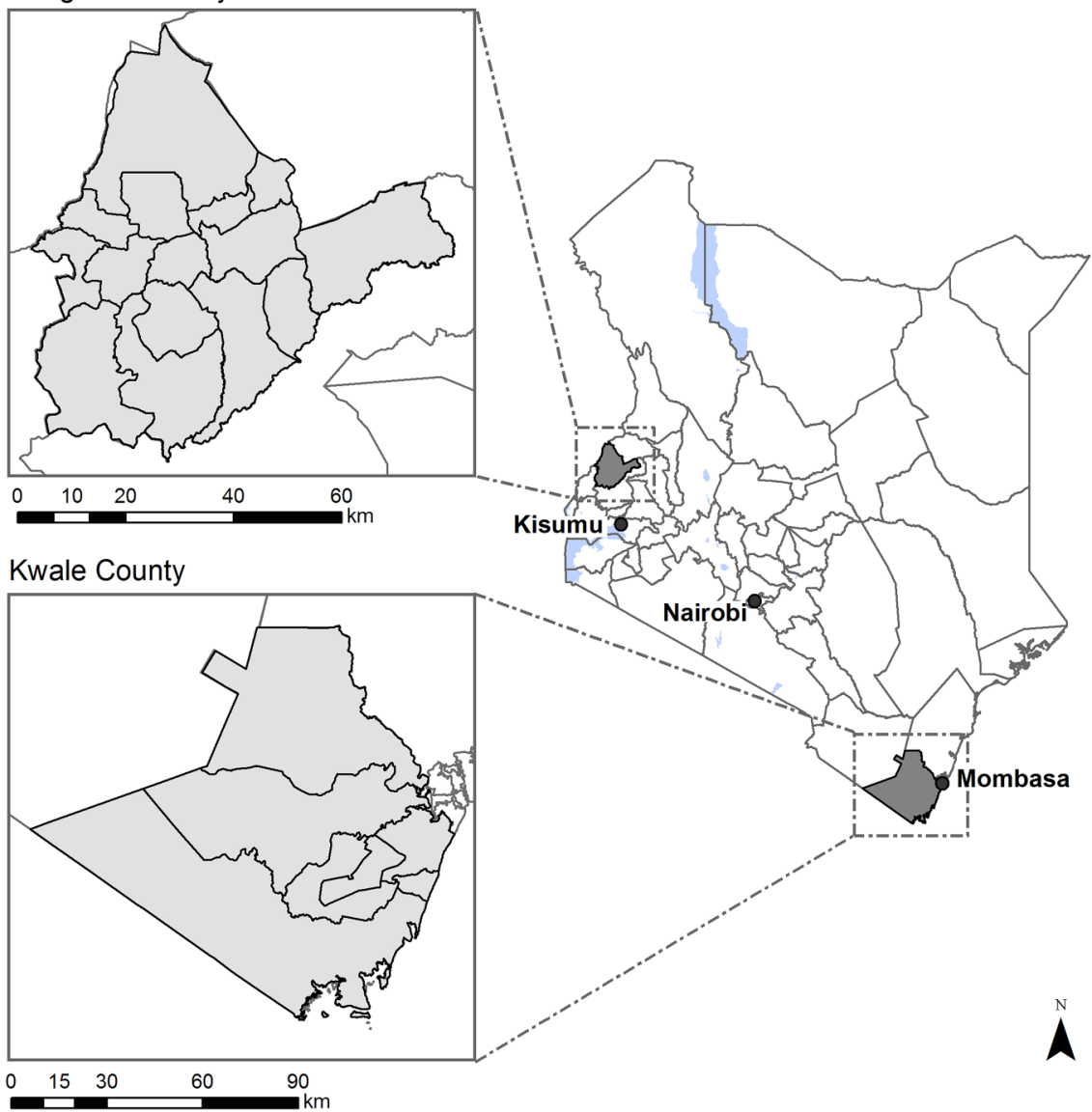

preschool-age and school-age children. ${ }^{8}$ Partners at the Paris meeting also committed support for evaluating the potential of interrupting the transmission of STHs using new tools and strategies. Recent analyses based on mathematical models of parasite transmission and the impact of treatment have suggested that the transmission of STHs can be interrupted (a breakpoint in transmission is crossed where parasite elimination is achieved) if treatment is expanded to adults and provided more frequently. ${ }^{9-11}$ While such models can provide new insights, there is an obvious need to test the predictions of the impact and cost-effectiveness of alternative treatment strategies through rigorous field studies.

If there is to be a move towards broadening the range of age groups targeted by STH treatment programmes, then it is necessary to identify suitable delivery systems. Possibly the longest running community-based NTD control programme that treats across all age groups is the African Programme for Onchocerciasis Control, which has helped countries create a community-directed treatment strategy by involving community-directed drug distributors and extending and strengthening health systems. ${ }^{12-14}$ A community-based approach is also employed by national lymphatic filariasis (LF) control programmes, whereby community drug distributors or community health workers (CHWs) provide communitywide delivery of albendazole plus ivermectin (or diethylcarbamazine in areas not endemic for onchocerciasis) to entire populations aged 2 years and above. ${ }^{15}$ Although CHWs are increasingly used to promote healthy behaviours and deliver basic health services, especially in poor and underserved communities, ${ }^{16}{ }^{17}$ the benefits of using a CHW-based approach for STH control is poorly understood at present.

This paper describes two cluster randomised trials in Kenya that seek to provide new evidence on the impact and cost-effectiveness of alternative treatment strategies and delivery systems in reducing the transmission of STHs. Such evidence will help establish proof-of-concept of the possibility of interrupting STH transmission and would likely be of value to policymakers in STH-endemic countries, and partners and funders supporting STH control.

\section{Aims and objectives}

The overall aim of the trials is to evaluate the impact and cost-effectiveness of school-based versus communitybased deworming on measures of STH transmission in Kenya. Specifically, we will test the hypothesis that treatment needs to be provided to a broad range of ages and/or at more frequent intervals than 1 year in order to interrupt the transmission of STH. The study also includes process and economic evaluations to assess the feasibility and implementation of the alternative 
Table 1 Epidemiological and socioeconomic characteristics in the two study areas

\begin{tabular}{|c|c|c|c|c|}
\hline & Bungoma & Kwale County & National average & Source \\
\hline \multicolumn{5}{|l|}{ Helminth infections } \\
\hline STHs combined (\%) & 49.3 & 33.6 & $32.4^{*}$ & 27 \\
\hline Hookworm (\%) & 44.3 & 27.7 & $15.6^{\star}$ & 27 \\
\hline Ascaris lumbricoides (\%) & 28.2 & 0.8 & $18.0^{*}$ & 27 \\
\hline Trichuris trichiura (\%) & 0.8 & 8.9 & $6.6^{*}$ & 27 \\
\hline Schistosoma haematobium (\%) & non-endemic & 17.5 & $14.8^{*}$ & 27 \\
\hline Wuchereria bancrofti (\%) & non-endemic & endemic & endemic in 6 of 47 counties & 28 \\
\hline \multicolumn{5}{|l|}{ Socioeconomic conditions } \\
\hline Poverty rate $(\%) \dagger$ & 52.2 & 72.9 & 46.6 & 29 \\
\hline \multicolumn{5}{|l|}{ Access to water and sanitation } \\
\hline Improved drinking water (\%) & 72.1 & 51.2 & 55.1 & 30 \\
\hline Improved sanitation (\%) & 71.2 & 34.4 & 64.9 & 30 \\
\hline \multicolumn{5}{|l|}{ School system } \\
\hline Primary school attendance (\%)‡ & 94.6 & 87.2 & 85.6 & 30 \\
\hline Literacy rate (\%) & 60.5 & 66.5 & 66.4 & 31 \\
\hline \multicolumn{5}{|l|}{ Health system } \\
\hline Full immunisation coverage (\%)§ & 84.4 & 77.5 & 83.0 & 32 \\
\hline Doctors (per 100000 people) & 4 & 1 & 7 & 32 \\
\hline Nurses (per 100000 people) & 37 & 37 & 49 & 32 \\
\hline
\end{tabular}

treatment strategies and delivery systems, which will guide scale-up of the programmes in Kenya and other settings in Africa. The more detailed study objectives are:

1. To quantify the impact of school-based versus community-based mass treatment (treatment strategies and delivery systems) at annual and biannual intervals (treatment strategies) in reducing the transmission of STH spp, hookworm, A. lumbricoides and T. trichiura.

2. To evaluate the costs and cost-effectiveness of alternative STH treatment strategies and delivery systems in reducing transmission.

3. To assess the extent to which community-based treatment programmes for STH are acceptable to the community, which are feasible, given the health system capacity, and can be easily scaled-up across Kenya and elsewhere.

\section{METHODS AND ANALYSIS}

Reporting of the study protocol has been verified in accordance with the SPIRIT (Standard Protocol Items for Randomised Trials) recommendations.

\section{Overall study design}

Two paired community cluster randomised trials in different settings in Kenya will evaluate the impact and cost-effectiveness of annual school-based deworming, annual community-based deworming and biannual community-based deworming. The trials are designed as cluster randomised, open-label trials with three study groups. The primary outcome is the prevalence of hookworm. This outcome was selected because it is the STH spp that contributes most to morbidity, is responsible for the most DALYs lost due to $\mathrm{STH}^{3}$ and is the species most difficult to control using school-based deworming alone. ${ }^{9}$ Allocation to study group is by cluster, using predefined units used in public health provision-termed community units (CUs). The three study groups are:

1. Control group: Annual school-based deworming. Preschool and school children (typically aged 2-14 years) will receive a single dose of albendazole $(400 \mathrm{mg})$ from trained school teachers, as part of the ongoing national school-based deworming programme.

2. Expanded age range group: Standard school-based deworming supplemented by annual communitybased deworming (2-99 years). All household members who are not enrolled in school will receive a single dose of albendazole (400 mg) from trained CHWs-known in Kenya as community health volunteers (CHVs).

3. Expanded age range and frequency group: Annual schoolbased deworming supplemented by community-based deworming (2-99 years), followed by an additional community-based deworming 6 months later. All household members who are not enrolled in school will receive a single dose of albendazole (400 $\mathrm{mg}$ ) from trained CHVs.

Mathematical models suggest that there is little difference in the impact of annual or biannual school-based 


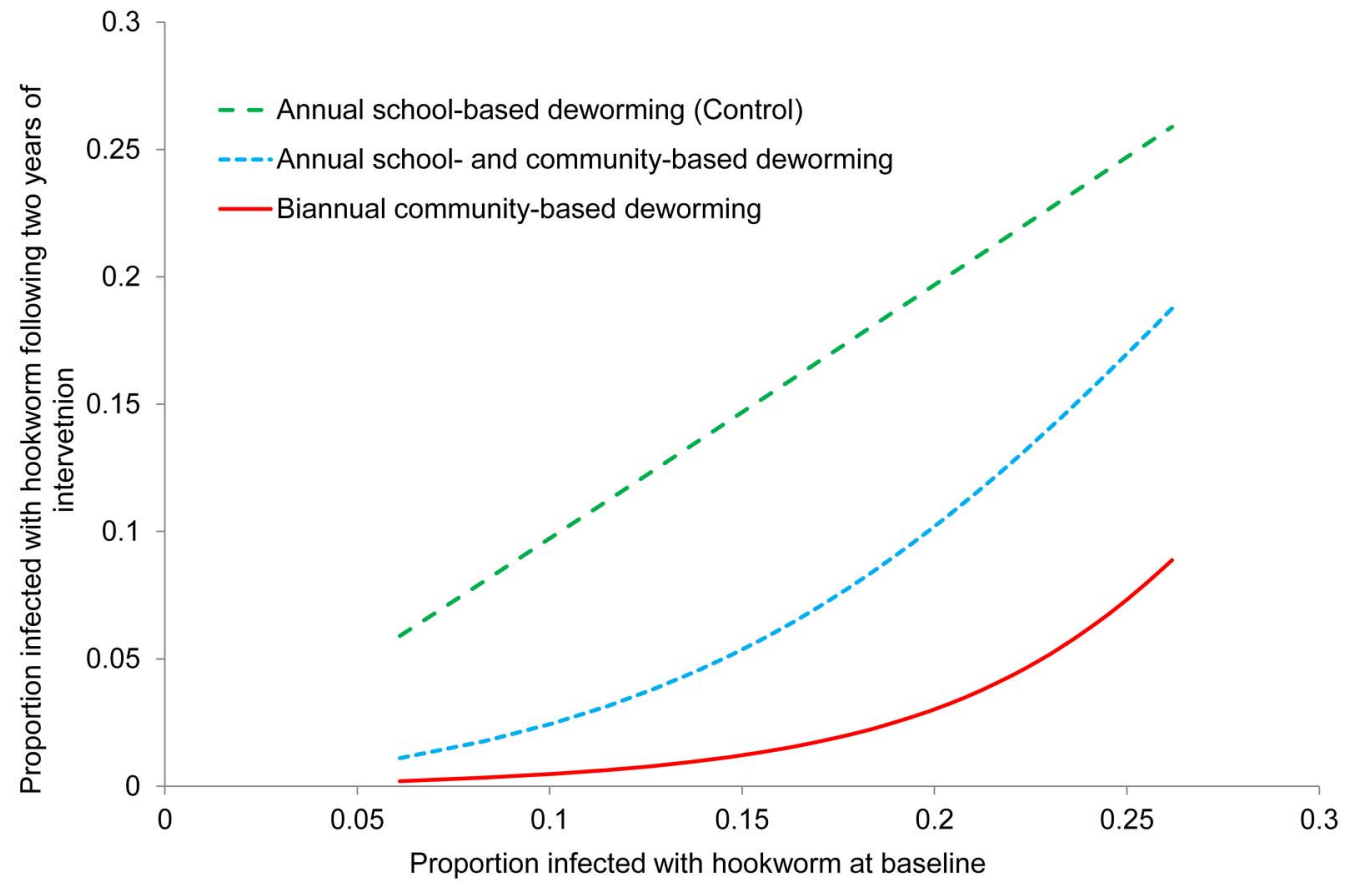

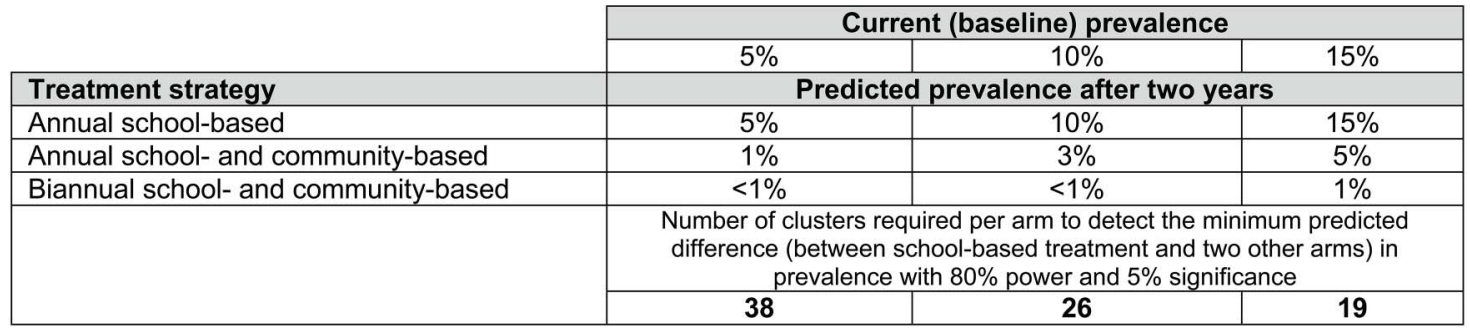

Figure 3 The relationship between baseline prevalence of hookworm infection (proportion of all community members found to be infected) and predicted impact following 2 years of treatment for each proposed treatment strategy. Based on a mathematical model of transmission dynamics, assuming $80 \%$ treatment coverage of school-based deworming and $70 \%$ of community-based treatment. Biannual school-based treatment did not differ significantly from annual school-based treatment and therefore is not shown. Sensitivity of diagnosis is assumed to be $63 \%$.

deworming, given the expected prevalence in our study sites, ${ }^{10} 18$ and therefore we did not include biannual school-based deworming as a study group. In other settings where A. lumbricoides is more common and hookworm is absent, biannual treatment may be desirable due to the relatively higher levels of infection in schoolage children compared to adults.

The primary outcome, the prevalence of hookworm infection, will be measured through cross-sectional parasitological surveys conducted at baseline and at 12, 24 and 30 months follow-up. The timing of the final follow-up survey takes into account differences in time since treatment of the annual and biannual treatment groups at 24 months. The overall study design is summarised in figure 1. A subsample of individuals from two CUs in each of the study groups will be followed longitudinally for two and half years, in order to better understand the transmission dynamics of STHs and to estimate key parameters for the mathematical models of transmission dynamics and treatment impact. A nested process evaluation, using semi-structured interviews, focus group discussions (FGDs) and a stakeholder analysis, will investigate the community acceptability, feasibility, given the local and regional health system structures and processes, and scale-up of the interventions.

\section{Interventions}

All study groups will receive treatment with albendazole (400 mg), which is highly efficacious against A. lumbricoides and hookworm, but has lower efficacy against T. trichiura. $^{19}$ What differs between the three study groups is the age range of populations that receive treatment and the frequency at which treatment is provided.

School-based deworming will be implemented in all communities as part of the national school-based deworming programme (NSBDP). Launched jointly in 2009 by the Ministry of Education, Science and Technology (MoEST), and the Ministry of Health $(\mathrm{MoH})$, and funded by the Children's Investment Fund Foundation, the programme's goal is to eliminate STHs and schistosomiasis as a national public health problem. A single $400 \mathrm{mg}$ dose of albendazole is provided 
annually by trained teachers on designated deworming days. The programme targets all children in at-risk subcounties enrolled in public and private primary schools and early childhood development centres (kindergartens). ${ }^{20}$ Non-enrolled school-age children are also encouraged to come to school for treatment on deworming day. The NSBDP makes use of a cascade model whereby trainings, materials, drugs and funds are channelled from the national level through the county to the school level, involving officials from MoEST and $\mathrm{MoH}$ throughout the cascade. The emphasis is on MoEST for delivery and MoH for supervision. Treatment coverage data and remaining drugs are returned along the reverse cascade. No adaptations shall be made to the NSBDP for the study other than modification of the treatment and coverage forms to strengthen the data capture on treatment at the individual level, and to validate treatment coverage.

Community-based deworming will be provided by CHWs, called CHVs in Kenya. CHVs already promote care seeking and compliance to antiretroviral and tuberculosis treatment, and mobilise populations during national health campaigns, as part of the national community health strategy. ${ }^{21}$ This strategy also includes the establishment of CUs, which each serve approximately 1000 households or 5000 people, with a single CHV providing service to approximately 100 households. For every 25 CHVs, there is one Community Health Extension Worker (CHEW) who is a Ministry of Health employee with training in public health or nursing. This CHEW provides supervision and technical support to the CHVs. The community health strategy was revised in 2010, with new guidance on the contents of CHV kits to include basic drugs such as paracetamol, albendazole and tetracycline. ${ }^{22}$ The community-based deworming in the present trials will provide door-to-door treatment, drawing on the previous experience of the National Programme for Elimination of LF, which, to date, has implemented four rounds of mass treatment (2002, 2005, 2008 and 2011), but with variable levels of treatment coverage. ${ }^{23}$

\section{Setting}

The study will be conducted in two settings of Kenya that have contrasting epidemiological and programmatic characteristics: Kwale County on the south Kenyan coast and Bungoma County in western Kenya (figure 2). Key epidemiological and sociodemographic characteristics of the two study areas are summarised in table 1 . Historically, STH infections have been highly prevalent in both regions but recent control efforts have reduced levels of infection. A. lumbricoides is more common in western Kenya, whereas hookworm predominates on the coast. ${ }^{20}$ T. trichiura is present in both settings, but at low levels. In Kwale County, in addition to STHs, there is focal transmission of Schistosoma haemotobium, and LF is endemic. $^{24-26}$ In relation to sociodemographic characteristics, Kwale County is among the poorest in
Kenya, with low levels of access to water and sanitation, and minimal primary school enrolment, while Bungoma lies close to national averages for these factors.

\section{Randomisation}

Allocation to study group will be by cluster, using CUs. In areas where there are no formal CUs in existence, we worked with the public health officers and CHEWs to delineate CUs in order to ensure that every village and government is assigned to a CU. Randomisation was stratified by the prevalence of hookworm (below and above $20 \%$ prevalence, as determined in the baseline survey), and subcounty and size (below and above 840 households), in order to reduce the likelihood of chance imbalances. Randomisation took place at public ceremonies. The randomisation sequence generation was undertaken by an independent statistician using computerised random number generation. Sealed envelopes containing CU identification were placed in prestratified ballot boxes, with delegates invited to select envelopes from the boxes and directed to put the selected envelope in a box labelled A, B or C (corresponding to the three study groups trial) according to the pregenerated randomisation sequence for that stratum. In each $\mathrm{CU}, 225$ households are randomly selected and one randomly selected household member is recruited into the cross-sectional surveys.

Owing to the nature of the interventions, participants are not blinded to their group randomisation. However, the identity of the study groups remains hidden until the completion of community sensitisation and randomisation to eliminate participation bias. In addition, the laboratory technicians conducting stool examinations and the statistician responsible for analysis are blinded to the group assignment.

Contamination between clusters may occur when people from one cluster receive treatment implemented in another cluster or have lower exposure to STH infection due to lower transmission in another cluster. Our use of CUs, which comprised groups of villages rather than single villages, helps reduce the possibility of contamination. CUs, including CUs located in urban and periurban areas, which generally are not comprised of distinct groups of villages, were excluded.

\section{Sensitisation and recruitment}

Key stakeholders and policymakers have been involved in the study and its design from conceptualisation. Meetings have been held with the MoH and MoEST in Nairobi, and at each study site, where key stakeholders were sensitised about the study objectives, intervention and evaluation procedures, and requested to provide input. Community meetings were held to describe the purpose of the study, the interventions, the evaluation procedures to be followed, and the risks and benefits of participation. Individuals had the opportunity to ask questions. Consent for the intervention was provided at the community level with the option for individuals to 
opt-out either from receiving treatment or study assessments.

Consent for the baseline and follow-up cross-sectional surveys is obtained at the individual level. Field staff enumerate all households through coordination with the chiefs and village elders. Subsequently, households selected for inclusion into the study are visited by field staff. In each household visited, written informed consent to conduct the household-level questionnaire is sought from the household head. Following this, a random function programmed in a smartphone selects the individual within the household who will provide a stool sample and answer further individual-level questions. Individual-level informed consent is sought from selected individuals (either for themselves or their children) and written assent sought from children over 13 years of age. Inclusion criteria for the selection of individuals include: (1) resident for at least 12 months, (2) willingness of adult aged 18 years and above or parent/guardian to provide written informed consent, and (3) provision of written assent to participate from children aged between 13 and 17 years. Exclusion criteria include: (1) recent (<12 months) resident or visitor to household at time of household visit, (2) refusal of informed consent and (3) refusal to assent by children aged $13-17$ years.

\section{Outcomes}

The primary outcome is the prevalence of hookworm (Necator americanus or Ancylostoma duodenale) infection among all sampled individuals during 30 months of follow-up. Owing to ethical considerations of treating those found infected during surveys, new populations of individuals will be selected for each cross-sectional survey (baseline, 12, 24 and 30 months). All participants are asked to provide a stool sample, which is examined in duplicate using the Kato-Katz method. Individuals found infected are revisited by the study team and treated with albendazole. In a random subset of individuals, additional confirmatory diagnosis of infection is based on real-time PCR, which also allows the differentiation between hookworm spp. ${ }^{33} 34$

The main secondary outcomes include:

- Prevalence of A. lumbricoides and T. trichiura, based on expert microscopy and, in a random subsample, on real-time PCR.

- Intensity of infection for each STH spp, based on quantitative egg counts.

- Treatment coverage, measured using both routine data, and data collected during household visits to track treatment coverage and compliance.

\section{Survey procedures}

At each house visited, household heads are interviewed to collect a household census and information on household characteristics and ownership of key assets during household visits. Data on household water, sanitation and hygiene (WASH) conditions and school WASH conditions are collected using structured observations and questionnaires, employing tools piloted and extensively used in Kenya. ${ }^{35} 36$

Teachers and CHVs will be provided with treatment registers and asked to provide a full record of all individuals who have received treatment. To augment these data, population-based coverage surveys using multistage clustering sampling ${ }^{37}$ will be carried out among a random subsample of communities.

In each cross-sectional survey, a randomly individual selected is asked to provide a stool sample, which is transported to a nearby health facility laboratory and examined in duplicate within $1 \mathrm{~h}$ of processing using the Kato-Katz method. Duplicate slides are read by independent microscopists. A $10 \%$ quality control check is performed by a supervisor. Aliquots of randomly selected stool samples are preserved in ethanol for confirmatory real-time PCR diagnosis. ${ }^{33} 34$ In addition, stool samples will be stored for future molecular analysis, including the detection of potential drug resistance alleles, ${ }^{35} 38$ and genome sequence analysis to investigate the genetic structure of helminth populations. ${ }^{39}$

All members of the study teams have been appropriately trained in the study objectives and procedures. Standard operating procedures have been developed, field-tested and revised, and are used to guide all field activities. Supervisors make regular visits to the field to monitor fieldwork.

\section{Longitudinal studies}

In six CUs (one with a medium (20-49\%) and one high ( $>50 \%)$ prevalence of hookworm in each study group), individuals will be followed longitudinally to help quantify the transmission dynamics of the parasites and re-parameterise mathematical models of transmission dynamics. An age-stratified random sample of 200 individuals will be chosen and asked to provide complete stool samples for a period of 5 days immediately following treatment. The collected stool will be transported to an off-site sorting facility, A. lumbricoides and hookworm will be manually separated from the stool, and the number and sex or worms recorded for each individual. The proportion of unfertilised eggs will also be determined for A. lumbricoides, given the importance of this measure as a determinant of how effective a given treatment programme is in driving transmission to extinction by crossing the transmission threshold where insufficient mating occurs to sustain transmission. ${ }^{40}$ Selected individuals will be revisited at 1, 3, 6, 9 and 12 months posttreatment and asked to provide a stool sample, which will be examined for the presence of STH eggs in duplicate using the Kato-Katz method.

We will also conduct household visits to assess the extent of non-compliance to treatment and factors associated with non-compliance. ${ }^{37}$ Adverse events will be monitored in these cohorts during household visits. Reports of severe adverse events that are classified as at 
least possibility related to the study drugs will be reported to the ethics committee.

\section{Sample size calculation}

Sample size calculations are based on the principles of cluster randomised trials. ${ }^{41}$ Analysis of survey data collected as part of the ongoing monitoring and evaluation of the NSBDP suggests that the prevalence of hookworm, the primary outcome, varies between 5\% and $10 \%$, with an intracluster correlation coefficient (ICC) of $0.125 .^{2}$ The assumed difference between the study groups in the prevalence of hookworm after 30 months is based on mathematical modelling of the predicted impact of different treatment strategies, ${ }^{11} 12$ and is presented in figure 3 for different degrees of prevalence of infection at baseline. The figure also presents the number of clusters required to detect the smallest predicted difference between school-based treatment and two other arms with $80 \%$ power a $5 \%$ level of significance, an ICC of 0.125 and 225 participants per cluster. Based on these calculations and taking into account potential loss to follow-up of CUs (assumed to be 5\%), a conservative sample size of 40 clusters per group will be used in both study areas.

\section{Data management}

Data are collected in the field using Samsung GT7552 smartphones running android operating system V.4.2. The questionnaires are programmed using Survey CTO software (http://www.surveycto.com) and data are downloaded daily using secure Wi-Fi in the field office into a web-based database. Backup of the database to a central server is performed daily. Laboratory results are recorded in laboratory books by technicians and doubleentered into a customised database and saved on a centralised server.

\section{Data analysis}

Primary analysis will be carried out on groups as randomised (intention-to-treat). Results will be presented as appropriate effects sizes with a measure of precision (95\% CIs). Clustering by CU will be included in all analyses. Our main analysis of the primary outcome, the prevalence of hookworm, and other secondary outcomes, will be based on cross-sectional analyses comparing the outcome at 30 months follow-up between study groups. Unadjusted and adjusted results will be presented for all analyses. Covariates in adjusted analyses will be specified a priori and will include subcounty and urban/rural classification, household socioeconomic status, and access to adequate water and sanitation. For continuous outcomes, analyses will adjust for baseline by inclusion of the cluster mean of the outcome in question as a covariate in statistical models. For binary outcomes (notably the primary outcome), no baseline adjustment will be made because of issues relating to the non-collapsibility of ORs.
Demographic and other baseline characteristics of clusters will be compared to check for imbalances between study groups. Tabulation of these measures will be generated using the intention to treat data sets. No significance tests will be performed to investigate for differences between groups at baseline. Where imbalances are suspected, further exploratory adjusted analyses will be carried out that include additional adjustment for these factors. Formal statistical testing will be restricted to comparison between the two community-based treatment groups and the school-based treatment group (control). A small number of secondary outcomes will be prespecified for statistical testing along with the primary outcome. No formal adjustment will be made for multiple testing but the number of outcomes formally tested will be restricted to fewer than 10 and the results interpreted with due caution. This includes multiplicity associated with the two experimental arms. No formal comparisons will be made between the two experimental arms as the study has not been powered for this analysis.

A small number of secondary subgroup analyses will be specified in advance and will be carried out using formal statistical tests for interactions. These will include household poverty, remote households, frequent noncompliers of treatment, non-enrolled children, and households without access to adequate water and sanitation. These analyses will help understand the impact heterogeneity of the interventions.

\section{Process evaluation}

A key study objective is to understand lessons for the scale-up of community-based deworming in Kenya and elsewhere in Africa. Therefore, a qualitative evaluation will seek to identify and describe key assumptions and conditions underlying the implementation, sustainability and scaling-up of the different strategies and delivery systems. The focus of the evaluation will centre on whether CHVs can be utilised for the effective delivery of chemotherapy for control of STH and what factors influence the use of CHVs, including what type of incentives, if any, should be given. Investigation will focus on: (1) community acceptability, which will be assessed during FGDs and in-depth interviews (IDIs); and (2) feasibility, including a situation and stakeholder analysis of the structural, organisational and management factors that enhance or constrain effective implementation. ${ }^{42}$ A series of FGDs will be conducted with community members, teachers, CHVs, CHEWs and local health officials, to better understand the acceptance and implementation of the interventions, using predefined and structured topic guides. FGDs will be stratified by location and socioeconomic status, and specific efforts will be made to reach groups that may be marginalised due to their economic and sociocultural position. IDIs will be carried out with a range of actors and opinion leaders in order to understand the process and constraints of the different delivery systems. The number of 
IDIs will depend on when saturation is reached, but they will include members of the county, and national health and education teams.

FGDs and interviews will be digitally recorded, with notes additionally taken, transcribed and translated. Transcripts will be imported into NVivo (QSR International, Doncaster, Australia), coded by two independent coders, and analysed using content analysis to identify emerging themes. ${ }^{43}$ Following descriptive analysis, patterns and linkages among views, experiences and behaviours of the participants will be explored. The collected data will provide important contextual information and a basis for evaluating the generalisability of the study findings. The work will benefit from previous qualitative evaluations by the research teams in the study areas. $^{44-47}$

\section{Cost analysis}

Cost data will be collected following an ingredients approach, based on a semistructured questionnaire and by consultation of the programme accounting system. Data collection will be based on a standardised costing framework, capturing fixed and recurrent costs incurred at school and community levels. The questionnaire will include both cash and in-kind contributions, and will be used to estimate financial and economic costs of the alternative treatment strategies (annual vs biannual deworming) and delivery systems (through schools by teachers and through door-to-door delivery by CHVs). Financial costs capture actual expenditures in terms of programme implementation, whereas economic costs include opportunity costs of teachers, CHVs and other stakeholders in delivering deworming. Opportunity costs of the government staff and community members will be calculated using local pay scales. Capital costs will be annuitised over the useful life of equipment, vehicles and other assets using a discount rate of $3 \%$. Costs will be assessed from a societal perspective. Analysis of costs will be linked to volume of treatment in order to determine cost functions. The work will also present an analysis of the full cost of running national programmes in Kenya. Itemised-costing and sensitivity analysis will enable estimation of the costs of scaled-up implementation, and implementation in settings with different epidemiological and programmatic characteristics.

\section{Mathematical modelling and cost-effectiveness analysis}

The questions being addressed in the trial arose from analyses of the predicted impact of mass treatment based on mathematical models of the transmission dynamics and control of STHs. ${ }^{9-11} 48$ The potential for transmission of STHs and other helminths in a defined setting can be quantified by the value of the basic reproductive number $\left(\mathrm{R}_{0}\right)$, which is defined as the average number of offspring produced by one female worm that survives to reproductive maturity. $\mathrm{R}_{0}$ can be stratified by age (age-related exposure), and various environmental and behavioural factors. ${ }^{49}$ In endemic communities, mathematical models of helminths that are dioecious have two stable points (no parasites and a stable endemic equilibrium of parasite persistence), separated by an unstable point-the so called 'breakpoint' in transmission (a point at which $\mathrm{R}_{0}$ falls to just above but close to unity (1) in value), below which continued treatment can quickly drive the population to extinction. For attempts at elimination, the goal of mass treatment is to drive the parasite population below the 'breakpoint' by treating sufficient fractions of the target population. Models of STH transmission provide insight into optimal treatment strategies for achieving this breakpoint, ${ }^{9} 10$ and show that annual or biannual community-based deworming can reduce overall prevalence and associated intensity substantially. As an example, reaching the transmission breakpoint can be achieved after 2 years given a baseline prevalence of $10 \%$ or less (low transmission setting) if coverage is high. The models also highlight how the age-distribution of worm burden determines the breadth of age groups that should be treated and the importance of considering the species mix.

Data arising from the trials will be used to validate the initial model predictions and to provide better estimates of key epidemiological parameters for use in model predictions. Specifically, the study will produce better estimates of the following parameters: density dependent fecundity, parasite distributions in the various age groupings (estimates of the negative binomial aggregation parameter k), age dependent exposure to infection, drug efficacy and treatment compliance. The models will be fitted with estimates of age-stratified patterns of reinfection and intensity of infection, as well as estimates of treatment coverage using Monte Carlo Markov Chain methods. Such model fitting and parameter estimation will allow examination of whether the observed and predicted impact is consistent. A stochastic model of STH transmission is also under development to enable estimates of variability around deterministic predictions (such as time to crossing the breakpoint) to be made taking account of the many heterogeneities involved in parasite transmission.

The models will be used to explore the impact of the different treatment strategies in a range of settings, with different underlying intensity in levels of transmission and treatment coverage, and the potential impact of alterations (in coverage and frequency) to the treatment strategies over time. The duration of treatment required to cross the 'breakpoint' in transmission will also be examined. Particular attention will be given to noncompliers to treatment (both persistent and irregular) in all study groups, and work will examine the impact on overall transmission of poor adherence to treatment.

The cost data, detailed above, will be integrated into the models for cost-effectiveness analysis. The incremental costs per infection, heavy infection, anaemia case and disability adjusted life years averted will be estimated for the two new strategies compared to the current situation with annual school-based deworming. Sensitivity analysis will be 
undertaken to account for uncertainties in the analysis, with attention given to the impact of non-compliance.

\section{ETHICS AND DISSEMINATION}

Ethical approval

The study has been approved by the Kenya Medical Research Institute and National Ethics Review Committee (SSC Number 2826) and the London School of Hygiene and Tropical Medicine (LSHTM) Ethics Committee (7177). Sponsorship and insurance is provided by the LSHTM's Clinical Trials Sub-Committee (QA615).

\section{Informed consent}

The study is intentionally embedded within the ongoing NSBDP, which will continue to deliver deworming to all schools in the study areas. At the time of household visits, household members are asked to give their verbal consent for their participation in the community-based deworming. Written informed consent is obtained from adults and parents or guardians of children, before enrolment in the cross-sectional surveys and longitudinal surveys. Written informed consent will also be sought from individuals included in the qualitative evaluations, including FGDs and in-depth interviews. Participants of FGDs and interviews will be provided the options not to be quoted in any reporting of findings. Study information sheets are provided in English, Kiswahili, Mijikenda, Luhya or Bukusu. Translated documents were verified through back-translation into English. Written assent to participate in the cross-sectional and longitudinal studies is obtained from children aged 13 years and above.

Risks and benefits of participating in the study are presented during community meetings and any issues arising discussed. The risk of participating in the trial is very low. The study drug, albendazole, is extremely safe and no severe adverse events are expected. ${ }^{50}$ In the unlikely situation of events occurring, these will be reported to the study site investigator and the principal will inform the ethics committee. Collection of stool samples is a routine procedure and is considered not to be a medical risk; there is the possibility of embarrassment, which will be minimised by appropriate action.

All information will remain confidential. Laboratory specimens, reports, data collection, and process and administrative forms will be identified by a coded unique identifier to maintain participant confidentiality. Access to collected data will initially be limited to fieldworkers at the point of data collection, and to the study statistician and investigators during analysis. As indicated below, data that are considered non-sensitive and do not include identifying participant information will be made publicly available once the main findings have been published, subject to appropriate data sharing agreements.

\section{Trial oversight}

No data safety and monitoring board will be established since the interventions are extremely safe and already delivered to hundreds of millions of individuals each year as part of national deworming programmes. Instead, an independent Data Monitoring Committee (DMC), consisting of a chair and three members, and operating under a remit of a charter, has been established to monitor data for quality and completeness. The DMC will review, in strict confidence, an interim analysis of the 12-month data. The DMC will also review and approve the final data analysis plan.

\section{Dissemination}

The study has a dedicated web page, on the Global Atlas of Helminth Infection (GAHI) website (http:// www.thiswormyworld.org/tumikia-project), where study updates and final results will be disseminated. Study findings will also be disseminated through multiple and innovative media, ensuring that research is presented in ways that are most appropriate for the various stakeholders identified during the stakeholder mapping. The data collected in the study along with the study instruments will be made publicly available following the publication of the main study findings, based on approved data sharing agreements.

\section{Author affiliations}

${ }^{1}$ Faculty of Infectious and Tropical Diseases, London School of Hygiene \&

Tropical Medicine, London, UK

${ }^{2}$ Eastern and Southern Africa Centre of International Parasite Control, Kenya Medical Research Institute, Nairobi, Kenya

${ }^{3}$ Evidence Action, Nairobi, Kenya

${ }^{4}$ Neglected Tropical Diseases Unit, Division of Communicable Disease Prevention and Control, Ministry of Health, Nairobi, Kenya

${ }^{5}$ Office of the Executive Committee, Medical Services and Public Health, Kwale County Government, Kwale, Kenya

${ }^{6}$ Faculty of Medicine, Department of Infectious Disease Epidemiology, London Centre for Neglected Tropical Disease Research, School of Public Health, St

Mary's Campus, Imperial College London, London, UK

${ }^{7}$ Warwick Mathematics Institute, University of Warwick, Coventry, UK

${ }^{8}$ School of Life Sciences, University of Warwick, Coventry, UK

${ }^{9}$ Faculty of Public Health and Policy, London School of Hygiene \& Tropical Medicine, London, UK

${ }^{10}$ Department of Environmental Health, Rollins School of Public Health, Emory University, Atlanta, Georgia, USA

${ }^{11}$ Faculty of Epidemiology and Population Health, London School of Hygiene \& Tropical Medicine, London, UK

Twitter Follow the Global Atlas of Helminth Infection at @ThisWormyWorld, Dina Balabanova at @dinabalabanova

Acknowledgements The authors sincerely thank the public health officers, public health technicians, community health extension workers, chiefs, assistant chiefs, village elders and nyuma kumi heads in Kwale County, for working tirelessly with us to delineate the community units and to compile the database of households in Kwale County. The authors are grateful to the communities for their very welcoming and cooperative response to the activities conducted thus far. The authors would also like to thank Stella Kepha, Leah Musyoka, Mary Wanjiru, Lennie Ngau, Harris Fondo, Steve Okiya, Kathryn Shuford, Jorge Cano, Alice Easton, Rita Oliveira, Mark Bradley, Donald Bundy, Julie Jacobson, Thomas Kisimbi, Karen Levy, Faith Rose, Sasha Zoueva, Alix Zwane, Grace Hollister, Karim Naguib, Rockie Jumapili, Redempta Muendo and the other members of the Kwale County Ministry of Health, and Ministry of Interior and Coordination of National Government for contributions to the study design, intervention implementation and/or study 
protocols. This paper is published with the permission of the Director of the Kenya Medical Research Institute.

Contributors SJB conceived the study, secured funding and drafted the manuscript. CSM, KEH, RLP and RMA additionally initiated the study design. CSM, SMN, PMG and JHK led the parasitology. DA, AC, CSM, PMG, CG-C and BN helped with implementation. HCT, JET and TDH provided modelling expertise. BW, DN and DB led the process evaluation. UKG and HCT led the economic evaluation. MCF led the assessment of water and sanitation. EA provided statistical expertise. All the authors contributed to the refinement of the study protocol and approved the final manuscript.

Funding The study is supported by the Bill and Melinda Gates Foundation (\#OPP1033751) and a grant from the DFID/MRC/Wellcome Trust global health trials scheme (MR/M021289/1), with additional funding provided by the Children's Investment Fund Foundation (CIFF). SJB is supported by a Wellcome Trust Senior Fellowship in Basic Biomedical Science (098045), which also supports RLP and KEH. GlaxoSmithKline, the manufacturer of albendazole, has donated, through the Government of Kenya, the drug used in the study.

\section{Competing interests None declared.}

Ethics approval Kenya Medical Research Institute and National Ethics Review Committee; London School of Hygiene and Tropical Medicine.

Provenance and peer review Not commissioned; peer reviewed for ethical and funding approval prior to submission.

Data sharing statement The data collected in the study will be publicly distributed along with metadata and survey instruments following publication of the primary results, which is expected to occur 24 months after the final data collection date, subject to approved data sharing agreements.

Open Access This is an Open Access article distributed in accordance with the terms of the Creative Commons Attribution (CC BY 4.0) license, which permits others to distribute, remix, adapt and build upon this work, for commercial use, provided the original work is properly cited. See: http:// creativecommons.org/licenses/by/4.0/

\section{REFERENCES}

1. World Health Organization. Accelerating work to overcome the global impact of neglected tropical diseases-a roadmap for implementation. Geneva: World Health Organization, 2012.

2. Murray CJ, Vos T, Lozano R, et al. Disability-adjusted life years (DALYs) for 291 diseases and injuries in 21 regions, 1990-2010: a systematic analysis for the Global Burden of Disease Study 2010. Lancet 2012;380:2197-223.

3. Baker MC, Krotki K, Sankara DP, et al. Measuring treatment coverage for neglected tropical disease control programs: analysis of a survey design. Am J Epidemiol 2013;178:268-75.

4. World Health Organization. Helminth control in school-age children. Geneva: World Health Organization, 2011.

5. Brooker S, Kabatereine NB, Fleming F, et al. Cost and costeffectiveness of nationwide school-based helminth control in Uganda: intra-country variation and effects of scaling-up. Health Policy Plan 2008;23:24-35.

6. Leslie J, Garba A, Oliva EB, et al. Schistosomiasis and soiltransmitted helminth control in Niger: cost effectiveness of school based and community distributed mass drug administration [corrected]. PLoS Negl Trop Dis 2011;5:e1326.

7. [No authors listed]. Soil-transmitted helminthiases: number of children treated in 2013. Wkly Epidemiol Rec 2015;10: 89-94.

8. Bill \& Melinda Gates Foundation. Global partners are taking the "neglect" out of "neglected tropical diseases". 2014. http://www. gatesfoundation.org/Media-Center/Press-Releases/2014/04/GlobalPartners-Are-Taking-the-Neglect-out-of-Neglected-Tropical-Diseases (accessed 28 May 2014)

9. Anderson RM, Truscott JE, Pullan RL, et al. How effective is school-based deworming for the community-wide control of soil-transmitted helminths? PLoS Negl Trop Dis 2013;7:e2027.

10. Anderson R, Truscott J, Hollingsworth TD. The coverage and frequency of mass drug administration required to eliminate persistent transmission of soil-transmitted helminths. Philos Trans $R$ Soc Lond B Biol Sci 2014;369:20130435.
11. Truscott JE, Hollingsworth TD, Brooker SJ, et al. Can chemotherapy alone eliminate the transmission of soil transmitted helminths? Parasit Vectors 2014;7:266.

12. Amazingo U. The African Programme for Onchocerciasis Control (APOC). Ann Trop Med Parasitol 2008;102(Suppl 1):19-22.

13. Traore MO, Sarr MD, Badji A, et al. Proof-of-principle of onchocerciasis elimination with ivermectin treatment in endemic foci in Africa: final results of a study in Mali and Senegal. PLoS Negl Trop Dis 2012;6:e1825.

14. Meredith SE, Cross C, Amazigo UV. Empowering communities in combating river blindness and the role of NGOs: case studies from Cameroon, Mali, Nigeria, and Uganda. Health Res Policy Syst 2012;10:16.

15. Global Programme to Eliminate Lymphatic Filariasis. Progress report 2000-2009 and strategic plan 2010-2020 of the global programme to eliminate lymphatic filariasis: halfway towards eliminating lymphatic filariasis. Geneva: World Health Organization, 2011.

16. Mwinzi PN, Montgomery SP, Owaga CO, et al. Integrated community-directed intervention for schistosomiasis and soil transmitted helminths in western Kenya-a pilot study. Parasit Vectors 2012;5:182.

17. Omedo MO, Matey EJ, Awiti A, et al. Community health workers' experiences and perspectives on mass drug administration for schistosomiasis control in western Kenya: the SCORE Project. Am J Trop Med Hyg 2012;87:1065-72.

18. Das JK, Salam RA, Arshad A, et al. Community based interventions for the prevention and control of Non-Helmintic NTD. Infect Dis Poverty 2014;3:24.

19. Keiser J, Utzinger J. Efficacy of current drugs against soil-transmitted helminth infections: systematic review and meta-analysis. JAMA 2008;299:1937-48.

20. Pullan RL, Gething PW, Smith JL, et al. Spatial modelling of soil-transmitted helminth infections in Kenya: a disease control planning tool. PLoS Negl Trop Dis 2011;5:e958.

21. Kenya Ministry of Health. Taking the Kenya essential package for health to the community: a strategy for the delivery of level one services. Nairobi: Ministry of Health, 2006.

22. Kenya Ministry of Health. Director of public health and sanitation: policy shift on community strategy. Nairobi: Ministry of Public Health and Sanitation, 2011.

23. Njenga SM, Mwandawiro CS, Wamae CN, et al. Sustained reduction in prevalence of lymphatic filariasis infection in spite of missed rounds of mass drug administration in an area under mosquito nets for malaria control. Parasit Vectors 2011;4:90.

24. Marchal B, Van Dormael M, Pirard M, et al. Neglected tropical disease (NTD) control in health systems: the interface between programmes and general health services. Acta Trop 2011;120(Suppl 1):S177-85.

25. Kihara J, Mwandawiro C, Waweru B, et al. Preparing for national school-based deworming in Kenya: the validation and large-scale distribution of school questionnaires with urinary schistosomiasis. Trop Med Int Health 2011;16:1326-33.

26. Hong ST, Chai JY, Choi MH, et al. A successful experience of soil-transmitted helminth control in the Republic of Korea. Korean $J$ Parasitol 2006;44:177-85.

27. Mwandawiro CS, Nikolay B, Kihara JH, et al. Monitoring and evaluating the impact of national school-based deworming in Kenya: study design and baseline results. Parasit Vectors 2013;6:198.

28. Cano J, Rebollo MP, Golding N, et al. The global distribution and transmission limits of lymphatic filariasis: past and present. Parasit Vectors 2014;7:466.

29. Kenya National Bureau of Statistics. Kenya integrated household budget survey, 2005-2006. 2006. https://opendata.go.ke/ (accessed 22 May 2015 2014)

30. Kenya National Bureau of Statistics. Population and Housing Census. 2009. http://www.knbs.or.ke/ (accessed 14 Mar 2014).

31. Commission of Revenue Allocation. Kenya County Factsheets. Kenya Open Data. 2011. https://opendata.go.ke/ (accessed 23 Oct 2014).

32. Kenya Ministry of Health. Kenya County Health Fact Sheets. 2013. http://www.healthpolicyproject.com/ (accessed 23 Oct 2014).

33. Verweij JJ. Application of PCR-based methods for diagnosis of intestinal parasitic infections in the clinical laboratory. Parasitology 2014;141:1863-72.

34. Verweij JJ, Stensvold CR. Molecular testing for clinical diagnosis and epidemiological investigations of intestinal parasitic infections. Clin Microbiol Rev 2014;27:371-418.

35. Freeman MC, Clasen T, Brooker SJ, et al. The impact of a school-based hygiene, water quality and sanitation intervention on soil-transmitted helminth reinfection: a cluster-randomized trial. Am J Trop Med Hyg 2013;89:875-83. 
36. Garn JV, Greene LE, Dreibelbis R, et al. A cluster-randomized trial assessing the impact of school water, sanitation, and hygiene improvements on pupil enrollment and gender parity in enrollment. J Water Sanit Hyg Dev 2013;3, 10.2166/ washdev.2013.217

37. Pullan RL, Brooker SJ. The global limits and population at risk of soil-transmitted helminth infections in 2010. Parasit Vectors 2012;5:81

38. Freeman MC, Ogden S, Jacobson J, et al. Integration of water, sanitation, and hygiene for the prevention and control of neglected tropical diseases: a rationale for inter-sectoral collaboration. PLOS Negl Trop Dis 2013;7:e2439.

39. Pullan RL, Smith JL, Jasrasaria $\mathrm{R}$, et al. Global numbers of infection and disease burden of soil transmitted helminth infections in 2010 . Parasit Vectors 2014;7:37.

40. Njomo DW, Mukoko DA, Nyamongo NK, et al. Increasing coverage in mass drug administration for lymphatic filariasis elimination in an urban setting: a study of Malindi Town, Kenya. PLOS ONE 2014;9: e83413.

41. Meheus F, Rijal S, Lutumba P, et al. NTD control and health system strengthening. Lancet 2012;379:2149-50.

42. Bleakley $\mathrm{H}$. Disease and Development: Evidence from Hookworm Eradication in the American South. Q J Econ 2007;122:73-117.
43. Pope C, Ziebland S, Mays N. Qualitative research in health care. Analysing qualitative data. BMJ 2000;320:114-16.

44. Jones CO, Wasunna B, Sudoi R, et al. "Even if you know everything you can forget": health worker perceptions of mobile phone text-messaging to improve malaria case-management in Kenya. PLOS ONE 2012;7:e38636.

45. Njomo DW, Amuyunzu-Nyamongo M, Magambo JK, et al. The role of personal opinions and experiences in compliance with mass drug administration for lymphatic filariasis elimination in Kenya. PLOS ONE 2012;7:e48395.

46. Okello G, Ndegwa SN, Halliday KE, et al. Local perceptions of intermittent screening and treatment for malaria in school children on the south coast of Kenya. Malar J 2012;11:185.

47. Wasunna B, Zurovac D, Goodman CA, et al. Why don't health workers prescribe ACT? A qualitative study of factors affecting the prescription of artemether-lumefantrine. Malar J 2008;7:29.

48. Anderson R, Hollingsworth TD, Truscott $\mathrm{J}$, et al. Optimisation of mass chemotherapy to control soil-transmitted helminth infection. Lancet 2012;379:289-90.

49. Anderson RM, Medley GF. Community control of helminth infections of man by mass and selective chemotherapy. Parasitology 1985;90:629-60.

50. Horton J. Albendazole: a review of anthelmintic efficacy and safety in humans. Parasitology 2000;121(Suppl):S113-32. 